\title{
How to find conductance tensors of quantum multi-wire junctions through static calculations: application to an interacting $\mathrm{Y}$ junction
}

\author{
Armin Rahmani, ${ }^{1}$ Chang-Yu Hou, ${ }^{2,3}$ Adrian Feiguin, ${ }^{4}$ Claudio Chamon, ${ }^{1}$ and Ian Affleck ${ }^{5}$ \\ ${ }^{1}$ Department of Physics, Boston University, Boston, MA 02215 USA \\ ${ }^{2}$ Department of Physics, Boston University, Boston, Massachusetts 02215 USA \\ ${ }^{3}$ Instituut-Lorentz, Universiteit Leiden, P.O. Box 9506, 2300 RA Leiden, The Netherlands \\ ${ }^{4}$ Department of Physics and Astronomy, University of Wyoming, Laramie, Wyoming 82071, USA \\ ${ }^{5}$ Department of Physics and Astronomy, University of British Columbia, \\ Vancouver, British Columbia, Canada, V6T $1 Z 1$
}

(Dated: May 14, 2018)

\begin{abstract}
Conductance is related to dynamical correlation functions which can be calculated with timedependent methods. Using boundary conformal field theory, we relate the conductance tensors of quantum junctions of multiple wires to static correlation functions in a finite system. We then propose a general method for determining the conductance through time-independent calculations alone. Applying the method to a $\mathrm{Y}$ junction of interacting quantum wires, we numerically verify the theoretical prediction for the conductance of the chiral fixed point of the Y junction and then calculate the thus far unknown conductance of its $\mathrm{M}$ fixed point with the time-independent density matrix renormalization group method.
\end{abstract}

Advances in molecular electronics can extend the limits of device miniaturization to the atomic scales where entire electronic circuits are made with molecular building blocks [1]. Single molecule junctions connected to two macroscopic metallic leads have already been successfully fabricated [2]. A key ingredient of any such molecular circuit is a junction of three or more quantum wires where electrical current is conducted through a molecular structure between the wires. Determining the conductance of such junctions is a long-sought and challenging goal.

Landauer-Büttiker's formalism does not account for electron-electron interactions which play a key role in low dimensions. Functional renormalization group methods have been helpful in studying the interaction effects [3] but they are also dependent upon the presence of noninteracting leads. There are a number of challenging problems in junctions of multiple interacting wires. For instance, a time-reversal symmetric Y junction of Luttinger liquids has a nontrivial fixed point known as the M fixed point [4], which has remained an open quantum impurity problem. For spinfull electrons, there are nontrivial fixed points even for junctions of two quantum wires [5]. Numerical methods like density matrix renormalization group (DMRG) 6, 7] could potentially be efficient tools for computing the conductance of junctions with an arbitrary number of wires and interactions. Conductance is a property of an open quantum system and is related to dynamical correlation functions. It may thus appear that one needs the more computationally demanding dynamical methods such as time-dependent DMRG for conductance calculations [8 14]. These require large systems and averaging over a time window.

The goal of this Letter is to find generic relations that permit the computation of conductance from static equilibrium calculations alone, thus making it possible to obtain the conductance tensors of arbitrary complex junc- tions with any number of leads using time-independent methods, such as standard DMRG. As a concrete application, we are interested in the open problem of the conductance of the $\mathrm{M}$ point of a $\mathrm{Y}$ junction of quantum wires. We argue that the $T=0$ linear conductance $G_{i j}$ (defined by $I_{i}=\sum_{j} G_{i j} V_{j}$ where $V_{j}$ is the voltage applied to wire $j$ and $I_{j}$ is the inward directed current on it) is determined by the conformally invariant boundary condition (BC) describing the infrared fixed point of quantum junctions. Finding the conductance could then be helpful in determining nontrivial fixed points.

Using boundary conformal field theory, we find a generic relationship between the dynamical correlation functions of a semi-infinite quantum junction and the static correlation functions of a finite system obtained by a conformal transformation. This relationship allows us to extract certain coefficients from a time-independent numerical calculation of the ground state expectation values of appropriate local operators which uniquely determine the dynamical correlation functions and give us the linear-response conductance of the junction through the Kubo formula. After establishing the generic continuum formalism, we discuss the application of the formalism to a discrete lattice computation.

We present DMRG results on the application of the method to an interacting $\mathrm{Y}$ junction of quantum wires. We successfully verify the theoretical prediction [4] of $G_{12}=-2 \frac{g}{3+g^{2}}(g+1) \frac{e^{2}}{h}$ for the chiral fixed point of a $\mathrm{Y}$ junction and numerically calculate the conductance at two values of the Luttinger parameter $g$ for the timereversal symmetric $\mathrm{M}$ fixed point.

Consider $M$ semi-infinite wires connected to a junction with arbitrary structure and interactions. We represent the system as a quasi-one-dimensional structure with all the wires running parallel to the positive $x$ axis (Fig. 1 left panel). The wires are connected to the molecular 
structure of the junction at $x=0$. Setting the velocity of the charge carriers to unity, we introduce the complex coordinates $z=\tau+i x$, where $\tau$ is the imaginary time. The multiple wires are described by $M$ species of electrons living on the upper half complex plane. Notice that spin can also be taken into account by doubling the number of species, but here we focus on spinless electrons.

The low energy behavior of the system is given by a renormalization group fixed point. Here we treat the junction problem as a boundary conformal field theory [15]. In the bulk of the wires, we have a conformal field theory (CFT) with central charge $M$ and bosonic fields $\phi_{j}, j=1, \ldots, M$ obtained from the fermionic fields via standard bosonization. We assume that the effect of the junction (the molecular structure connecting the wires) on the fixed-point behavior is generically encoded in a conformally invariant boundary condition for the CFT in the upper half-plane and represented by a boundary state $|\mathcal{B}\rangle$ [16]. This assumption is at the heart of a paradigm in quantum impurity problems and has been repeatedly verified in a multitude of such problems [17.

Let us first consider the CFT in the absence of the boundary. In terms of the bosonic fields $\phi_{j}$ or their dual fields $\theta_{j}$, the action for this system is given by

$$
S=\sum_{j} \frac{g}{4 \pi} \int d^{2} x \partial_{\mu} \phi^{j} \partial^{\mu} \phi^{j}=\sum_{j} \frac{1}{4 \pi g} \int d^{2} x \partial_{\mu} \theta^{j} \partial^{\mu} \theta^{j}
$$

where $g$ is the Luttinger parameter. For noninteracting wires $g=1$ and we have $g<1(g>1)$ for repulsive (attractive) interactions. The primary operators for this CFT are the vertex operators $e^{i \alpha \phi_{j}}$ (the fermion creation and annihilation operators are of this form) and the currents

$$
J_{L}^{j}(z)=\frac{i}{\sqrt{2} \pi} \partial \theta^{j}(z, \bar{z}) \quad J_{R}^{j}(\bar{z})=-\frac{i}{\sqrt{2} \pi} \bar{\partial} \theta^{j}(z, \bar{z})
$$

where $\partial \equiv \partial_{z}=\frac{1}{2}\left(\partial_{\tau}-i \partial_{x}\right)$ and $\bar{\partial} \equiv \partial_{\bar{z}}=\frac{1}{2}\left(\partial_{\tau}+\right.$ $\left.i \partial_{x}\right)$. In the absence of a boundary, the only nonvanishing correlation functions are the chiral ones:

$$
\left\langle\mathcal{T}_{\tau} J_{L}^{i}\left(z_{1}\right) J_{L}^{j}\left(z_{2}\right)\right\rangle=-\frac{g}{4 \pi^{2}} \frac{\delta_{i j}}{\left(z_{1}-z_{2}\right)^{2}}
$$

and similarly for $\left\langle\mathcal{T}_{\tau} J_{R}^{i}\left(\bar{z}_{1}\right) J_{R}^{j}\left(\bar{z}_{2}\right)\right\rangle$ where $\mathcal{T}_{\tau}$ indicates imaginary time-ordering.

Let us now consider the system on the upper halfplane. In general, the presence of the boundary does not change the correlation between primary operators of the same chirality [18] but introduces additional correlations between the left movers and the right movers,

$$
\left\langle\mathcal{T}_{\tau} J_{L}^{i}\left(z_{1}\right) J_{R}^{j}\left(\bar{z}_{2}\right)\right\rangle=-\frac{g}{4 \pi^{2}} A_{\mathcal{B}}^{i j} \frac{1}{\left(z_{1}-\bar{z}_{2}\right)^{2}} .
$$

The coefficients $A_{\mathcal{B}}^{i j}$ are determined by the $\mathrm{BC}$ on the real axis. In fact, in terms of the boundary state $|\mathcal{B}\rangle$, we have $A_{\mathcal{B}}^{i j}=\frac{\left\langle J_{L}^{i} J_{R}^{j}, 0 \mid \mathcal{B}\right\rangle}{\langle\mathbb{1}, 0 \mid \mathcal{B}\rangle}$ where $|O, 0\rangle$ is the highest weight state corresponding to a generic operator $O$ (here $\left.O=J_{L}^{i} J_{R}^{j}\right)[19]$. Note that at the fixed point the correlators are determined by conformal symmetry up to the coefficients above and as long as we are interested in the fixed-point conductance of a given junction, a direct numerical calculation of dynamical correlation functions is not required. The fact that the dynamical correlations are directly related to equal-time correlations by conformal invariance is the key reason why we can obtain the conductance from a time-independent calculation.

If we know the coefficients $A_{\mathcal{B}}^{i j}$, we can use the Kubo formula [4]

$$
G_{i j}=\lim _{\omega \rightarrow 0_{+}}-\frac{e^{2}}{\hbar} \frac{1}{\omega L} \int_{-\infty}^{\infty} d \tau e^{i \omega \tau} \int_{0}^{L} d x\left\langle\mathcal{T}_{\tau} J^{i}(y, \tau) J^{j}(x, 0)\right\rangle
$$

with $J^{j}=J_{R}^{j}-J_{L}^{j}$ to obtain the conductance of the junction upon performing the integrations. Let us focus on calculating the off-diagonal elements of the conductance tensor $G_{i j}$. The diagonal elements are not independent since $\sum_{i} G_{i j}=\sum_{j} G_{i j}=0$ due to current conservation and the fact that a common voltage applied to all wires gives zero current. For $i \neq j$, as seen in Eq. (11), the correlators of the same chirality vanish. Using the Kubo formula above and after doing the $\tau$ integral by contour integration we obtain for $i \neq j$

$$
\begin{aligned}
G_{i j} & =g \frac{e^{2}}{h} \frac{1}{L} \int_{0}^{L} d x\left[A_{\mathcal{B}}^{i j} H(x+y)+A_{\mathcal{B}}^{j i} H(-x-y)\right] \\
& =A_{\mathcal{B}}^{i j} g \frac{e^{2}}{h}
\end{aligned}
$$

where $H$ is the Heaviside step function. Since both $x$ and $y$ are positive, $H(-x-y)=0$ in the second term.

The next task is to extract $A_{\mathcal{B}}^{i j}$ by measuring an equaltime ground state correlation function. The conductance is well-defined for a semi-infinite system, while the equilibrium equal-time correlators are easily obtainable in a finite system. To get around this issue, we use the following conformal transformation to map the upper halfplane $z=\tau+i x$ into a strip of width $\ell$,

$$
w=\frac{\ell}{\pi} \ln z, \quad z=e^{\frac{\pi}{\ell} w} .
$$

Notice that for $w=u+i v$ we have $0<v<\ell$. The mapping takes the positive real axis to the boundary $v=$ 0 and the negative real axis to $v=\ell$.

Now consider a primary operator $O$ (here $O=J_{L}^{i} J_{R}^{j}$ ) with

$$
\langle O(z)\rangle=A_{\mathcal{B}}^{O}(2 x)^{-X_{O}}
$$

in the semi-infinite plane with $\mathrm{BC} \mathcal{B}$ on the real axis. Using the transformation $\langle O(w)\rangle=\left|\frac{d w}{d z}\right|^{-X_{O}}\langle O(z)\rangle$ we obtain after some algebra

$$
\langle O(w)\rangle=A_{\mathcal{B}}^{O}\left[2 \sin \left(\frac{\pi}{\ell} v\right) / \frac{\pi}{\ell}\right]^{-X_{O}}
$$



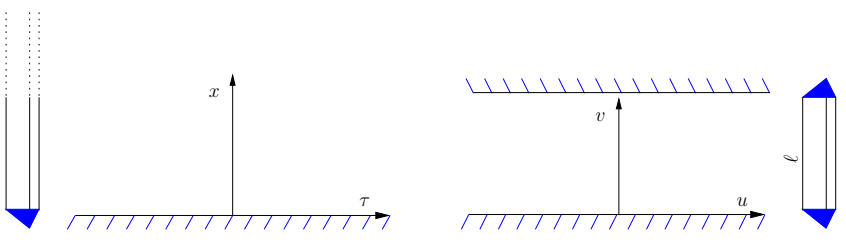

FIG. 1. The conformal mapping from the upper half-plane to the strip and the corresponding physical systems. The actual junction (here a $\mathrm{Y}$ junction, for instance) is illustrated next to to the corresponding complex plane regions. The left panel shows the semi-infinite junction (the upper half-plane) and the right panel shows the finite system obtained by the conformal mapping (the strip).

Since the zero temperature finite system is invariant under translations in $u$, the static ground state expectation value of the local operator $J_{L}^{i}(x) J_{R}^{j}(x)$ with $x$ the distance from a boundary in the finite system of length $\ell$ is expected to behave as $-\frac{g}{4 \pi^{2}} A_{\mathcal{B}}^{i j}\left[2 \sin \left(\frac{\pi}{\ell} x\right) / \frac{\pi}{\ell}\right]^{-2}$. This ground state expectation value permits the determination of the $A_{\mathcal{B}}^{i j}$ coefficients and thus the conductance via Eq. (2) using solely static equilibrium computations.

In the remainder of this Letter, we discuss the application of the method to a tight-binding lattice numerical calculation. First we consider the BCs for the finite system obtained from the transformation Eq. (3). Because we are assuming a conformally invariant $\mathrm{BC}$, the boundary $v=0$ will have the same $\mathrm{BC} \mathcal{B}$ as the real axis of the semi-infinite plane. To determine the $\mathrm{BC}$ for the $v=\ell$ boundary created by the transformation, we consider the fermion creation and annihilation operators which transform as

$\Psi_{L}(w)=\left(\frac{d w}{d z}\right)^{-\frac{1}{2}} \Psi_{L}(z), \quad \Psi_{R}(\bar{w})=\left(\frac{d \bar{w}}{d \bar{z}}\right)^{-\frac{1}{2}} \Psi_{R}(\bar{z})$.

Using $\frac{d \bar{w}}{d \bar{z}}=\left.\frac{d w}{d z}\right|_{v=0}=\frac{\ell}{\pi} e^{-\frac{\pi}{\ell} u}$ and $\frac{d \bar{w}}{d \bar{z}}=\left.\frac{d w}{d z}\right|_{v=\ell}=$ $-\frac{\ell}{\pi} e^{-\frac{\pi}{\ell} u}$, we find that up to a sign coming from choosing the branch of the square root in the transformation above, the $\mathrm{BC}$ at $v=\ell$ is the same as $v=0$. This does not mean however that in a microscopic implementation, one should place the mirror image of the junction at $v=\ell$. Notice that the chiral fields switch role for the two boundaries; i.e. the right-movers are the outgoing (incoming) states for the $v=0(v=\ell)$ boundary. So for instance if the $\mathrm{BC}$ at $v=0$ is described by an S-matrix $S$, the BC at $v=\ell$ is given by the inverse S-matrix $S^{\dagger}$ up to a sign.

We consider a half-filled system with an even number of sites in each wire and particle-hole symmetry in the bulk of the wires. The Hamiltonian of the system is given by $H_{L}+H_{B}+H_{R}$ where $H_{B}$ is the bulk Hamiltonian of the wires and $H_{L, R}$ are the junction Hamiltonians. In terms of the fermionic operators $\Psi_{m}^{j}$ on lead $j$ and site $m=1, \ldots l$, we have

$$
H_{B}=\sum_{j, m}\left[-\Psi_{m}^{j \dagger} \Psi_{m+1}^{j}+\text { h.c. }+V\left(n_{m}^{j}-\frac{1}{2}\right)\left(n_{m+1}^{j}-\frac{1}{2}\right)\right]
$$

where $n_{m}^{j} \equiv \Psi_{m}^{j \dagger} \Psi_{m}^{j}$, the hopping amplitude is set to unity and $V$ is the interaction strength. To implement the correct boundary condition at $v=\ell$, we construct $H_{R}$ by applying a particle-hole and a time-reversal transformation to $H_{L}$. As a concrete example we have the boundary contribution $H_{R}=-\Psi_{l}^{i \dagger} \Gamma_{i, j} \Psi_{l}^{j}$ if $H_{L}=\Psi_{1}^{i \dagger} \Gamma_{i, j} \Psi_{1}^{j}$ for a Hermitian matrix $\Gamma$.

The coefficients $A_{\mathcal{B}}^{i j}$ can be extracted from the ground state correlators of chiral operators in a finite system. We cannot directly model chiral fermionic creation and annihilation operators, but indeed we can model chiral currents using density and current operators. The (nonchiral) current operator on the link between sites $m$ and $m+1$ is given by $J_{m}^{j}=i\left(\Psi_{m+1}^{j^{\dagger}} \Psi_{m}^{j}-\Psi_{m}^{j^{\dagger}} \Psi_{m+1}^{j}\right)$. Also, for a bond between sites $m$ and $m+1$, the charge density operator (with the background charge subtracted) is given by $N_{m}^{j}=\frac{1}{2}\left(n_{m}^{j}+n_{m+1}^{j}-\left\langle n_{m}^{j}\right\rangle-\left\langle n_{m+1}^{j}\right\rangle\right)$. Chiral current operators on the lattice are related to the density and nonchiral current through

$$
J^{j}(x)=v\left(J_{R}^{j}(x)-J_{L}^{j}(x)\right), \quad N^{j}(x)=J_{R}^{j}(x)+J_{L}^{j}(x)
$$

where $v$ is the velocity of charge carriers, and $x$ takes values in the middle of the bonds, $x=m+1 / 2$.

The Luttinger parameter $g$ and the velocity $v$ depend on the strength of the interaction [20, 21]. We have from the Bethe ansatz

$$
g=\frac{\pi}{2 \arccos (-V / 2)}, \quad v=\pi \frac{\sqrt{1-(V / 2)^{2}}}{\arccos (V / 2)} .
$$

We would like to find the off-diagonal elements of the conductance tensor $G_{i j}$. The chiral correlators are proportional to $\delta_{i j}$ in the half-plane and therefore vanish for $i \neq j$. They also vanish in the finite system since it is obtained from the half-plane by a conformal mapping. Using Eq. (4), we can then write for $i \neq j$

$$
\begin{aligned}
\left\langle J^{i}(x) J^{j}(x)\right\rangle & =-v^{2}\left(\left\langle J_{L}^{i}(x) J_{R}^{j}(x)\right\rangle+\left\langle J_{R}^{i}(x) J_{L}^{j}(x)\right\rangle\right), \\
\left\langle N^{i}(x) J^{j}(x)\right\rangle & =v\left(\left\langle J_{L}^{i}(x) J_{R}^{j}(x)\right\rangle-\left\langle J_{R}^{i}(x) J_{L}^{j}(x)\right\rangle\right) .(6
\end{aligned}
$$

By measuring the above two correlation functions (the second correlator $\left\langle N^{i}(x) J^{j}(x)\right\rangle$ is identically zero in the absence of a magnetic flux), we can find the chiral correlators we need for calculating the conductance. We fit the measured $\left\langle J_{L}^{i}(x) J_{R}^{j}(x)\right\rangle$ with $\tilde{A}\left[2 \sin \left(\frac{\pi}{\ell} x\right) / \frac{\pi}{\ell}\right]^{-2}$ to find the coefficient $\tilde{A}$ and then obtain the conductance using Eq. 2. Note that the fixed point behavior cannot be observed close to the boundary so the numerical results for small $x$ must be excluded in fitting the data. 
Below we consider an interacting $\mathrm{Y}$ junction of three quantum wires. As a check, we first apply the method to the chiral fixed point whose conductance has been predicted in Ref. [4]. We then present the first numerical calculation of the conductance of the time-reversal symmetric $\mathrm{M}$ fixed point whose properties have so far remained unknown. As seen in the inset of Fig. 2, the junction simply connects the three endpoints of the wires with a hopping amplitude $t$ (the amplitude is 1 in the bulk). Assuming that the loop formed at the junction is threaded by a magnetic flux $\phi$, the system will renormalize to the chiral fixed point for nonzero $\phi$ and $1<g<3$ independently of the hopping amplitude $t$. In the same range of $g$, the system renormalizes to the $\mathrm{M}$ fixed point in the absence of a magnetic flux $(\phi=0)$.

The numerical calculations for both fixed points were performed for $g=1.5,2.0$ and $t=0.7,1.0$. For the chiral fixed point, using $\phi=\pi / 2, \ell=39$ (40 sites on each chain) and the number of DMRG states $m=600$, we found strong agreement between the numerics and the predicted conductance $G_{12}=-2 \frac{g}{3+g^{2}}(g+1) \frac{e^{2}}{h}[4]$ as seen in Fig. 2. The correlation functions are independent of the hopping amplitude $t$ indicating convergence to the universal conductance of the fixed point. For the $\mathrm{M}$ fixed point, using $\phi=0, \ell=59$ and $m=1000$, we obtain a conductance of $G_{12}=-0.5293 \frac{e^{2}}{h}\left(G_{12}=-0.6146 \frac{e^{2}}{h}\right)$ for $g=1.5(g=2.0)$. None of the properties of the M fixed point were previously known in the literature 17. These values of the conductance depend on $g$ nontrivially and are different from those of all the other fixed points which have been identified so far. Thus our method and the numerical results provide evidence that the $\mathrm{M}$ fixed point is of a different nature.

In summary, we presented in this Letter a generic relationship between the elements of the conductance tensor of quantum junctions at a renormalization group fixed point and coefficients in the static correlation functions of chiral current operators. The method hinges on a fundamental relationship between static and dynamic correlation functions in boundary conformal field theory. We proposed a method for obtaining conductances from static calculations by extracting these coefficients using nonchiral current and density correlations in a lattice computation. We applied the method to an interacting $\mathrm{Y}$ junction of Luttinger liquids using time-independent DMRG and presented a numerical verification of the theoretically predicted conductance of the chiral fixed point as well as a calculation of the previously unknown conductance of the M fixed point. We emphasize that this general method turns the time-independent DMRG into a powerful tool for extracting conductances of completely generic molecular electronic junctions which constitute a wide class of quantum impurity problems.

We are grateful to A. Sandvik for helpful discussions. This work was supported in part by the DOE Grant No.

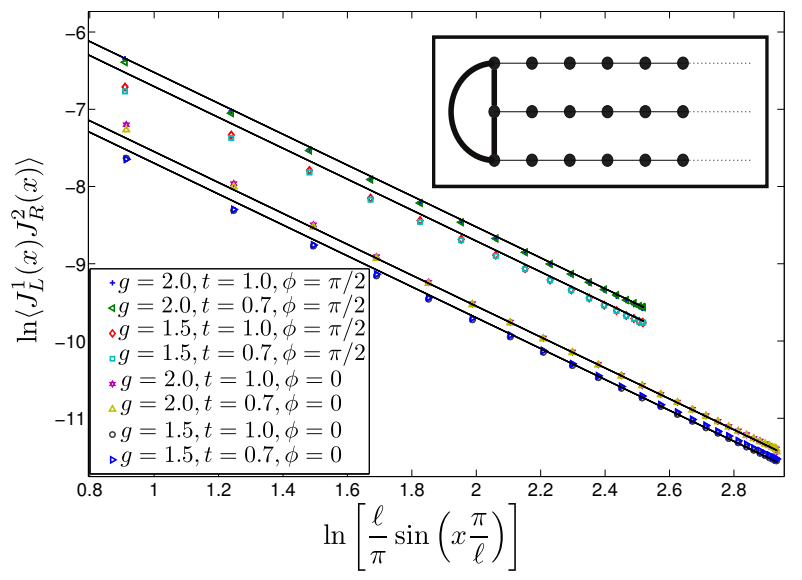

FIG. 2. The inset shows the lattice $Y$ junction. The thick bonds at the junction have a hopping amplitude $t$. The lines drawn through the data points show the CFT prediction with the exact analytical conductance in the case of the chiral fixed point $(\phi \neq 0)$ and the fit used for obtaining the conductance in the case of the $\mathrm{M}$ fixed point $(\phi=0)$. As expected, the conductance is independent of $t$.

DE-FG02-06ER46316 (CC, CH, and AR), NSF DMR0955707 (AF), NSERC (IA) and CIfAR (IA).

[1] A. Nitzan and M. A. Ratner, Science 300, 1384 (2003).

[2] M. A. Reed et al. Science 278, 252 (1997).

[3] X. Barnabe-Theriault et al., Phys. Rev. B 71, 205327 (2005); Phys. Rev. Lett. 94, 136405 (2005).

[4] C. Chamon, M. Oshikawa, and I. Affleck, Phys. Rev. Lett. 91, 206403 (2003) ; M. Oshikawa, C. Chamon, and I. Affleck, J. Stat. Mech. P02008 (2006); C.-Y. Hou and C. Chamon, Phys. Rev. B 77, 155422 (2008).

[5] C. L. Kane and M. P. A. Fisher, Phys. Rev. B 46, 15233 (1992).

[6] S. R. White, Phys. Rev. Lett. 69, 2863 (1992).

[7] H. Guo and S. R. White, Phys. Rev. B 74, 060401(R) (2006).

[8] M. A. Cazalilla and J. B. Marston, Phys. Rev. Lett. 88, 256403 (2002); See also 91, 049702 (2003).

[9] H. G. Luo, T. Xiang, and X. Q. Wang, Phys. Rev. Lett. 91, 049701 (2003).

[10] S. R. White, and A. E. Feiguin, Phys. Rev. Lett. 93, 076401 (2004); A. J. Daley, C. Kollath, U. Scholwök and G. Vidal, J. Stat. Mech.: Theor. Exp., P04005 (2004).

[11] P. Schmitteckert, Phys. Rev. B 70, 121302 (2004).

[12] K. A. Al-Hassanieh, A. E. Feiguin, J. A. Riera, C. A. Busser, E. Dagotto, Phys. Rev. B 73, 195304 (2006).

[13] F. Heidrich-Meisner, A. E. Feiguin, and E. Dagotto, Phys. Rev. B 79, 235336 (2009).

[14] L. Dias da Silva et al, Phys. Rev. B 78, 195317 (2008).

[15] J. L. Cardy, Nucl. Phys. B 324, 581 (1989).

[16] E. Wong and I. Affleck, Nucl. Phys. B 417, 403 (1994).

[17] I. Affleck, in Exact Methods in Low-Dimensional Statistical Physics and Quantum Computing (Ed: J. Jacobsen et al., Oxford University Press, Oxford, 2010), 3. 
[18] I. Affleck and A. W. W. Ludwig, Phys. Rev. B 48, 7297 (1993).

[19] J. L. Cardy and D. C. Lewellen, Phys. Lett. B 259, 274 (1991).
[20] K.-I. Imura, K.-V. Pham, P. Lederer, and F. Piéchon, Phys. Rev. B 66, 035313 (2002).

[21] T. Giamarchi, Quantum Physics in One Dimension (Oxford University Press, Oxford, 2003). 